\title{
Effective Crude Oil Pickering Emulsification by Oil-soluble Polymer Brush through Inverse Phase Emulsion Polymerization
}

\author{
Xiaohan Wang ${ }^{1}$, Zhiyuan Zheng ${ }^{1}$, Zhishuang $\mathrm{Ye}^{1}$, Xiaochi Liu ${ }^{1}$, Qingsong Yang ${ }^{1}$, Xuhong \\ $\mathrm{Guo}^{1}$, Jun $\mathrm{Xu}^{1}$, and Yisheng $\mathrm{Xu}^{1}$ \\ ${ }^{1}$ East China University of Science and Technology
}

May 5, 2020

\begin{abstract}
Various surfactants and amphipathic polymers have been utilized to enhance the flowability of crude oil. Alternatively, nanoparticulate polymer brushes serve as an ideal scaffold to form Pickering emulsions with crude oil due to its large specific area, excellent stability and tunable surface properties. In our research, a novel type of oil soluble polymer brushes was utilized to achieve stable crude oil emulsification by forming Pickering emulsions in water. Combining inverse emulsion polymerization and photo-emulsion polymerization, poly(N-Vinylcarbazole) (PVK) chains were successfully grafted onto the water soluble PAA core dispersed in hexane and the oil-soluble latex polymer brush features mono-dispersity, well-tuned size, fluorescence and stability. Significantly, the amphiphilic latex particles were successfully utilized to stabilize crude oil droplets in water by forming Pickering emulsions with apparent viscosity reduced by $99 \%$. These novel particles could serve as promising candidates to be applied in petrochemical industry by improving heavy crude oil flowability.
\end{abstract}

\section{INTRODUCTION}

Nowadays, crude oil serves as one of the most important natural resources for chemical engineering and energy supply. Due to extensive exploration, most of the current crude oil reserve remains as heavy oil with viscosity exceeding $10000 \mathrm{mPas}$ and the high viscosity of crude oil could pose severe challenges to its exploration, refinery and transportation process by forming conglomeration and congestion ${ }^{[1-2]}$. Therefore, it is of vital importance to reduce the viscosity of crude oil and improve its flowability for current chemical industries. Among various viscosity reduction practices, chemical additives serve as a promising solution due to its stable performance and cost efficiency. For example, researchers have widely used amphipathic polymers, such as $\alpha$-olefins-maleic anhydride copolymers ${ }^{[3-5]}$, ethylene-vinyl acetate copolymers ${ }^{[6]}$, and poly $[\mathrm{N}, \mathrm{N}-$ (dimethylamino) ethyl methacrylate] (PDMA)-poly(lauryl methacrylate) (PLMA) copolymers ${ }^{[7]}$ to stabilize crude oil in water by forming emulsions and the effect of internal and external parameters such as chain lengths, hydrophilicity/hydrophobicity balance as well as $\mathrm{pH}$ and ionic strength on emulsification process has been extensively investigated.

As an alternative to traditional emulsifiers, micro- or nanoparticles have been utilized to form Pickering emulsions due to its unparalleled strengths such as stability, tunability and stimuli-responsiveness ${ }^{[8-11]}$. As a result, it has been widely applied to advanced industrial and biomedical applications such as conducting biphasic catalysis ${ }^{[12-13]}$ and building compartmentalized artificial cells ${ }^{[14]}$. Moreover, researchers have relied on the unique properties of Pickering emulsions to promote crude oil emulsification process. For example, Saha et al. utilized carbon black (CB) particles with proper hydrophilic/hydrophobic balance to emulsify model oil and crude oil in water ${ }^{[15]}$. Compared with commercial surfactant, the CB particles are stable and biodegradable with their hydrophobicity easily tuned by adding acid or salt. However, nanoparticles are rarely used to stabilize crude oil and quantitative study of its emulsification effect on crude oil, as represented by viscosity or interfacial tension change, is lacked somehow in previous cases ${ }^{[15-16]}$. Due to the 
larger specific area as well as delicate and tunable structure, nanoparticles, especially amphipathic core-shell or Janus nanoparticles have great potential to improve crude oil flowability by forming Pickering emulsions. For example, poly(N-isopropylacrylamide) (PNIPAM) modified magnetic nanoparticles have been used to form oil-in-water Pickering emulsions for targeted oil separation ${ }^{[16]}$. After being exposed to an external magnetic, the latex particles could be efficiently separated from water and oil recovery could be achieved by elevating the temperature to dissemble the assembly. Recently, our group has conducted research on utilizing water-soluble spherical polymer brushes (SPB) to stabilize model oil, i.e., decane or hexadecane in water ${ }^{[17]}$. After introducing water-soluble polystyrene (PS)-poly(sodium p-styrenesulfonate) (PSS) brushes into oil/water mixtures, Pickering emulsions with well-tuned performance could be prepared successfully. However, in their cases, model oil including toluene and dodecane was used and the emulsification stability of crude oil in water still remains a challenge due to its high viscosity and complicated composition.

Herein, we present a novel practice to significantly enhance crude oil flowability and emulsion stability by forming Pickering emulsions in water via monodispersed oil-soluble spherical polymer brushes with high stability, which were prepared by inverse phase emulsion polymerization. The core and brush layer consisted of poly(acrylic acid) (PAA) and poly(N-Vinylcarbazole) (PVK) respectively and the length as well as photo-luminescence of the PVK chains could be tailored by the dose of $\mathrm{N}$-Vinylcarbazole $(\mathrm{VCz})$ with its nanostructure analyzed by small-angle X-ray scattering (SAXS). More importantly, utilizing the hydrophobicity of the brush layer and hydrophilicity of the core, efficient crude oil stabilization could be achieved by forming Pickering emulsion with viscosity lowered by $99 \%$ for over a month, which enables the nanoparticulate Pickering system to be a potential alternative of traditional dispersants in crude oil transportation and oil spillage remediation.

\section{RESULTS AND DISCUSSION}

\section{Characterization of oil soluble brush-like latex particles}

The schematic representation for the preparation of the oil-soluble PAA-PVK brushes is given in Figure 1. PAA latex particles were synthesized via inverse emulsion polymerization. The aqueous phase contained both acrylic acid and sodium acrylate, which was emulsified by Tween 80 and Span 80 in the oil phase to form $\mathrm{W} / \mathrm{O}$ emulsion under mechanical stirring. Hexane and toluene was chosen as the continuous phase due to its low viscosity and lipophilic nature. The polymerization of acrylic acid was initiated by thermo-initiator AIBN. At the end of the polymerization, photoinitiator HMEM was added in starving condition to ensure a thin layer of HMEM on the surface of PAA core by copolymerization with the residual AA monomers. Finally, the grafting photo-luminescent monomers, $\mathrm{VCz}$, were polymerized as chains affixed to the surface of PAA nanoparticles via photo emulsion polymerization, forming brush-like structure in oil-phase. The brush-like strucutre could endow the oil-soluble PAA-PVK latex nanoparticles with hydrophilicity in core layer and hydrophobicity in brush layer so it could be utilized to stabilize crude oil in water and reduce its viscosity by forming Pickering emulsions.

In previous reports ${ }^{[18]}$, monodispersed PAA core was synthesized in paraffin. However, utilizing paraffin as the reaction media suffered from many drawbacks such as high viscosity and difficulty to desiccate and characterize. Therefore, solvent applied to disperse and obtain PAA core was changed to hexane to make it readily for further processing. The size and size distribution of PAA core synthesized in hexane is shown in Figure S1.The hydrodynamic diameter $\left(D_{\mathrm{H}}\right)$ of PAA cores synthesized in hexane was to be around 100 $\mathrm{nm}$ and the optimal size distribution of PAA core synthesized in toluene and hexane fitted by Gaussian distribution is of a polydispersity index (PDI) below 0.01, demonstrating the PAA cores are mono-dispersed in hexane.

As polymer chains germinated from the surface of spherical PAA core via "grafting from" method, the hydrodynamic diameter increased and the growth of PVK brush layers could be monitored from the size varization by DLS. The size and size distribution of PAA core and PAA-PVK brushes with different monomer content was shown in Figure 2 (a). After grafting PVK chains through photo emulsion polymerization, the $D$ $\mathrm{H}$ of PAA-PVK brushes with $5 \%, 10 \%, 15 \%$ and $25 \% \mathrm{VCz}$ content exhibited marked increase from $100 \mathrm{~nm}$ 
to $132 \mathrm{~nm}, 164 \mathrm{~nm}, 192 \mathrm{~nm}$ and $238 \mathrm{~nm}$ respectively. Moreover, the layer thickness of PAA-PVK brushes, as determined by the size increase of the latex particles could be modulated by the dose of $\mathrm{VCz}$ monomer as shown in Figure 2 (b) and the monotonously increasing layer thickness with the $\mathrm{VCz}$ concentration could demonstrate that the PVK chains have been attached to the PAA core successfully. The polydispersity index of PAA core and PAA-PVK brushes of different $\mathrm{VCz}$ contents was $0.045,0.034,0.062,0.071,0.037$ respectively, which remained within monodisperse range before and after grafting. Moreover, the synthesized PAA core and PAA-PVK brushes could remain stable for over two months. As shown in Figure S2, thanks to the steric protection effect of PVK chains, the average size of PAA-PVK brushes doesn't exhibit marked increase even after storage for durable period. The oil-solubility of the obtained PAA-PVK brushes was tested subsequently. After desiccation, the PAA-PVK brushes could be redispersed in various organic solvent like toluene, dimethylformamide (DMF) and N-methyl pyrrolidone (NMP). According to the size distribution of PAA-PVK brushes redispersed in these organic solvents in Figure S3, the overall size of PAA-PVK brushes remained consistent after drying and redispersing in different lipophilic media and the slightly larger size and PDI compared to the original state could be ascribed to partial aggregation during the desiccation process. Furthermore, compared to PAA core, the size variation of PAA-PVK brushes during redispersion process remain less evident due to the protection of PVK layers. Therefore, the stable lipophilic dispersion of the as-synthesized PAA-PVK brushes could ensure stable performance for various applications, such as crude oil emulsification and storage of oil-soluble drugs.

To further verify the structures of the latex particles, the TEM images of as-synthesized PAA core and PAA-PVK brushes were obtained as shown in Figure 3. In Figure 3 (a) and (b), the PAA core was clearly identified with well-defined spherical shape with average diameter of $100 \mathrm{~nm}$ and the result was in good agreement with DLS analysis. After grafting PVK chains onto the surface, the overall distribution was relatively good without apparent aggregation as shown in Figure 3 (c) and from the enlarged image of PAAPVK brushes with higher resolution in Figure 3 (d), spherical shaped corona around the PAA core marked with red dotted circle could be clearly observed, indicating the existence of PVK brush layer around the PAA core. Moreover, measurements of the corona thickness in the dried state according to the TEM images reveal values of about $60 \mathrm{~nm}$, coinciding with the DLS results.

Since PVK, a photo-luminescent polymer was grafted onto polymer beads, the oil-soluble PAA-PVK brushes could exhibit well-tuned fluorescence property. The UV-vis and fluorescent spectra of PAA core and PAAPVK brushes dispersed in hexane is shown in Figure 4, in which the characteristic absorption peak at $328 \mathrm{~nm}$ and $340 \mathrm{~nm}$ and emission peak at $360 \mathrm{~nm}$ of PVK could be clearly observed for PAA-PVK brushes in both of the spectra. Moreover, with the increasing dose of polymerized $\mathrm{VCz}, \mathrm{PL}$ intensity of PAA-PVK brushes enhanced correspondingly, which could serve as another convincing evidence of successful grafting of PVK chains. Interestingly, maximum emission peak exhibited evident red shift with increasing $\mathrm{VCz}$ monomer dose, which might be attributed to $\pi-\pi$ stacking effect of the carbazole groups caused by the hydrophobic interaction between the polymer backbone of the grafted PVK chains ${ }^{[19-21]}$.

Emerging as a powerful characterization method to present structural information of macromolecules, small angle X-ray scattering (SAXS) has been widely used to provide detailed information about the nanostructure of nanoparticles ${ }^{[22-24]}$. Therefore, SAXS was also utilized to characterize the core-shell structure and size distribution of PAA-PVK brushes. The scattering intensity curves of PAA core and PAA-PVK brushes and the optimized fits of the corresponding distribution of excess electron density $\Delta \rho^{\mathrm{e}}(\mathrm{r})$ are displayed in Figure 5. As shown in Figure 5 (a), compared to PAA core, the scattering intensity of PAA-PVK brushes increased dramatically and the first maximum peak shifted to smaller q values, demonstrating the increased electron density due to the successful grafting of PVK chains onto the PAA core. Five-layer model was then chosen to analyze the structure of PAA-PVK brushes. From the radial distribution of excess electron density shown in Figure 5 (b), the average radius of PAA core and PAA-PVK brushes was about $34 \mathrm{~nm}$ and $108 \mathrm{~nm}$, which was slightly lower than the analyzing results of DLS. The difference could be attributed to the different mechanism of these two characterization methods. According to previous reports ${ }^{[25-27]}$, DLS is used for the detection of hydrodynamic diameter which is sensitive to the extension of exterior chains and determined by the longest chains of polymer brush while SAXS focuses on the average size calculated from statistical 
modeling analysis. Moreover, it is noteworthy that the dramatic decrease of excess electron density of the second layer could be clearly observed while the excess electron density declined slightly for outer layers, which differs from the gradual decreasing tendency observed in our previous studies of water-soluble SPBs by $\mathrm{SAXS}^{[23,28]}$. Such phenomenon could be ascribed to the distinctive structure of the PAA-PVK brushes. Due to the hydrophobic nature of the PVK chains, the oil-soluble PAA-PVK brushes dispersed in hexane lacked the electrostatic repulsion prevalent in traditional spherical polymer brushes dispersed in water. Hence, the PVK brush layer may exhibit a locally collapsed state around the PAA core with chains coiling and entangled with each other while the end of the chains could stretch in the outer space, leading to the uneven distribution of surface electrons. Therefore, judged from results by SAXS, we could conclude that PVK chains have been successfully grafted onto PAA core, forming distinctive brush structure. Moreover, to deal with further applications such as oil-soluble drug delivery and storage, the loading position and stability could be precisely monitored in situ by SAXS.

\section{Crude oil emulsification capacity of PAA-PVK latex particles}

Since the synthesized oil-soluble brushes feature a hydrophilic core and hydrophobic shell, we further investigated its application potential as a crude oil emulsifier. Crude oil emulsification is of vital importance for petro-chemical industry since it plays an important role in facilitating the collection and transportation process of crude oil. Besides various conventional surfactants, particles represent a promising avenue in realizing oil stabilization by forming Pickering emulsions due to its high stability and various functionalities ${ }^{[15]}$. After addition of a small amount of PAA-PVK brushes into the mixture of water and oil, both model oil and crude oil could disperse well in water and we could easily observe well defined oil-in-water emulsion droplets in the optical micrographes of Figure 6. The emulsion droplets for crude oil feature small and relatively uniform particles with average size as $3.2 \pm 0.7 \mu \mathrm{m}$ according to size analysis. Furthermore, although PAA core could also facilitate the formation of emulsion due to its partial amphipathy as shown in Figure S4, the PAA-PVK brushes could contribute to smaller latex particles with evidently narrower distribution by additional amphipathic effect compared to those formed in PAA core $(4.1 \pm 1.1 \mu \mathrm{m})$, and the same tendency could also be observed for model oil. Interestingly, after introducing normal water-soluble PS-PSS brushes of similar size (293 nm, the DLS data was shown in Figure S5) under the same procedures, the crude oil could not be dispersed into water as shown in Figure S6, probably due to the strong hydrophilicity of the shell layers. In previous cases ${ }^{[7]}$, the presence of too much polyelectrolyte chains would prevent surfactant from accumulating in oil-water interfaces due to electrostatic repulsion, so using oil-soluble polymer brushes we could ensure sufficient contact of crude oil and reduce the interfacial tension effectively.

To further quantify the emulsification effect of PAA-PVK brushes, we measured the apparent viscosity of emulsion solutions compromising crude oil, water and PAA-PVK brushes of different concentrations. According to rheology tests shown in Figure 7, the apparent viscosity of crude oil was significantly reduced after introducing PAA-PVK brushes and forming Pickering emulsions. After increasing the concentration of PAA core or PAA-PVK brushes, the viscosity of oil-in-water emulsions decreased monotonically and reached a plateau after reaching certain level. To be specific, at room temperature, the viscosity has declined by over $99 \%$ from $12200 \mathrm{mPas}$ for crude oil (Figure S7) to 623, 501.7, 141.2, 131.1, $116.2 \mathrm{mPas}$ after introducing PAA-PVK brushes of $2,4,6,8,10 \mathrm{mg} / \mathrm{mL}$ into the system while the emulsion viscosity under PAA core of the same concentration gradient was 1727, 702.4, 657.6, 438.1, $334.5 \mathrm{mPas}$. Evidently, the emulsification effect was significantly improved after grafting PVK brushes onto PAA core due to the additional amphiphilic effect, consistent with the observation of smaller and more uniform latex particles in optical micrograph of Figure 6. Moreover, the viscosity of crude oil emulsions formed in PAA-PVK brushes could remain stable within 30 days thanks to the high stability of Pickering emulsions while for PAA cores (Figure 8), phase separation between water and oil gradually happened after storing for 15 days with viscosity increasing dramatically. According to previous research ${ }^{[29-30]}$, the grafted polymers on the nanoparticulate brushes could contribute to higher dilatational modulus and could prevent the emulsions from coalescing and Ostwald ripening. So in this way, compared with bare particles, the nanoparticulate brushes could achieve better stability of Pickering emulsions. Therefore, the formation of stable oil-in-water emulsion facilitated by the oil soluble spherical polymer brushes could endow this novel polymer system with great potential in oil collection and 
transportation.

\section{CONCLUSION}

In summary, stable crude oil emulsification has been achieved firstly by oil-soluble nanoparticles. Combining inverse emulsion polymerization and photo emulsion polymerization, PVK chains with different lengths were successfully grafted onto the water soluble PAA core dispersed in hexane and the novel series of oil-soluble latex polymer brush features mono-dispersity, well-tuned size and fluorescence, multiple oil-solubility and stability. More importantly, the amphiphilic nature of the latex particles could be utilized to stabilize crude oil droplets in water by forming Pickering emulsions with viscosity reduced by $99 \%$ for 30 days. Therefore, the oil-soluble core-shell latex particles serve as promising candidates to be applied in petrochemical industry by improving heavy crude oil flowability during the exploration, transportation, and recovery process.

Supporting Information

Supporting Information is available online from Wiley InterScience or from the author.

Acknowledgements

The authors gratefully acknowledge the support of the National Natural Science Foundation of China (21306049), the Fundamental Research Funds for the Central Universities (222201314029), and the higher school specialized research fund for the doctoral program to this work (20110074110003).

Received: ((will be filled in by the editorial staff)) Revised: ((will be filled in by the editorial staff)) Published online: ((will be filled in by the editorial staff $)$ )

References

[1] Ge, J.; Shi, L.-A.; Wang, Y.-C.; Zhao, H.-Y.; Yao, H.-B.; Zhu, Y.-B.; Zhang, Y.; Zhu, H.-W.; Wu, H.-A.; Yu, S.-H., Nat. Nanotechnol. 2017, 12, 434.

[2] Hasan, S. W.; Ghannam, M. T.; Esmail, N., Fuel2010, 89 , 1095.

[3] Li, T.; Xu, J.; Zou, R.; Jiang, H.; Wang, J.; Li, L.; Cohen Stuart, M. A.; Prud'homme, R. K.; Guo, X., Ind. Eng. Chem. Res.2017, $56,12447$.

[4] Li, T.; Wang, T.; Xu, J.; Zou, R.; Si, Z.; Becker, J.; Li, L.; Cohen Stuart, M. A.; Prud'homme, R. K.; Guo, X., Ind. Eng. Chem. Res. 2018, 57, 4887.

[5] Li, L.; Xu, J.; Tinsley, J.; Adamson, D. H.; Pethica, B. A.; Huang, J. S.; Prud'homme, R. K.; Guo, X., Aiche J. 2012,58, 2254.

[6] Ashbaugh, H. S.; Guo, X.; Schwahn, D.; Prud'homme, R. K.; Richter, D.; Fetters, L. J., Energy Fuels 2005,19, 138 .

[7] Huang, J.; Xu, J.; Chen, K.; Wang, T.; Cui, C.; Wei, X.; Zhang, R.; Li, L.; Guo, X., Ind. Eng. Chem. Res. 2015,54, 1564 .

[8] Wu, J.; Ma, G.-H., Small 2016, 12 , 4633.

[9] Kim, K.; Kim, S.; Ryu, J.; Jeon, J.; Jang, S. G.; Kim, H.; Gweon, D.-G.; Im, W. B.; Han, Y.; Kim, H.; Choi, S. Q., Nat. Commun. 2017, $8,14305$.

[10] Tilton, R. D., Aiche J. 2019, 65, 3.

[11] Li, Q.; Huang, Y.; Xi, S.; Yang, Q.; Li, G., Aiche J.2013, 59 , 4373.

[12] Zhang, S.; Hong, B.; Fan, Z.; Lu, J.; Xu, Y.; Pera-Titus, M.,ACS Appl. Mater. Interfaces 2018, 10 , 26795 .

[13] Pera-Titus, M.; Leclercq, L.; Clacens, J.-M.; De Campo, F.; Nardello-Rataj, V., Angew. Chem. 2015, $54,2006$. 
[14] Liu, X.; Zhou, P.; Huang, Y.; Li, M.; Huang, X.; Mann, S.,Angew. Chem., Int. Ed. 2016, 55 , 7095.

[15] Saha, A.; Nikova, A.; Venkataraman, P.; John, V. T.; Bose, A.,ACS Appl. Mater. Interfaces 2013, 5 , 3094 .

[16] Chen, Y.; Bai, Y.; Chen, S.; Ju, J.; Li, Y.; Wang, T.; Wang, Q., ACS Appl. Mater. Interfaces 2014, 6 , 13334 .

[17] Zhang, Y.; Chen, K.; Cao, L.; Li, K.; Wang, Q.; Fu, E.; Guo, X., Polymers 2019, 11.

[18] Kriwet, B.; Walter, E.; Kissel, T., J. Control Release.1998, 56 , 149.

[19] Bai, L.; Wang, P.; Bose, P.; Li, P.; Zou, R.; Zhao, Y.,ACS Appl. Mater. Interfaces 2015, 7 , 5056.

[20] Qu, J.; Zhang, J.; Grimsdale, A. C.; Müllen, K.; Jaiser, F.; Yang, X.; Neher, D., Macromolecules 2004, 37,8297 .

[21] Rebarz, M.; Dalasiński, P.; Bała, W.; Łukasiak, Z.; Wojdyła, M.; Kreja, L., Opt. Appl. 2005, 35 , 407.

[22] Ballauff, M., Prog. Polym. Sci. 2007,32, 1135.

[23] Wang, W.; Li, L.; Henzler, K.; Lu, Y.; Wang, J.; Han, H.; Tian, Y.; Wang, Y.; Zhou, Z.; Lotze, G.; Narayanan, T.; Ballauff, M.; Guo, X.,Biomacromolecules 2017, 18 , 1574.

[24] Han, H.; Li, L.; Wang, W.; Tian, Y.; Wang, Y.; Wang, J.; von Klitzing, R.; Guo, X., Langmuir 2017, 33,9857 .

[25] De Robillard, Q.; Guo, X.; Ballauff, M.; Narayanan, T.,Macromolecules 2000, 33 , 9109.

[26] Dingenonts, N.; Patel, M.; Rosenfeldt, S.; Pontoni, D.; Narayanan, T.; Ballauff, M., Macromolecules 2004,37, 8152 .

[27] Yu, X. J.; Wang, W. H.; Li, L.; Guo, X. H.; Zhou, Z. M.; Wang, F. C., Chin. J. Polym. Sci. 2014, 32 , 778 .

[28] Tian, Y.; Li, L.; Han, H.; Wang, W.; Wang, Y.; Ye, Z.; Guo, X.,Polymers 2016, 8 .

[29] Alvarez, N. J.; Anna, S. L.; Saigal, T.; Tilton, R. D.; Walker, L. M., Langmuir 2012, 28 , 8052.

[30] Xie, G.; Krys, P.; Tilton, R. D.; Matyjaszewski, K.,Macromolecules 2017, 50 , 2942.

Schemes, Figures and Tables 


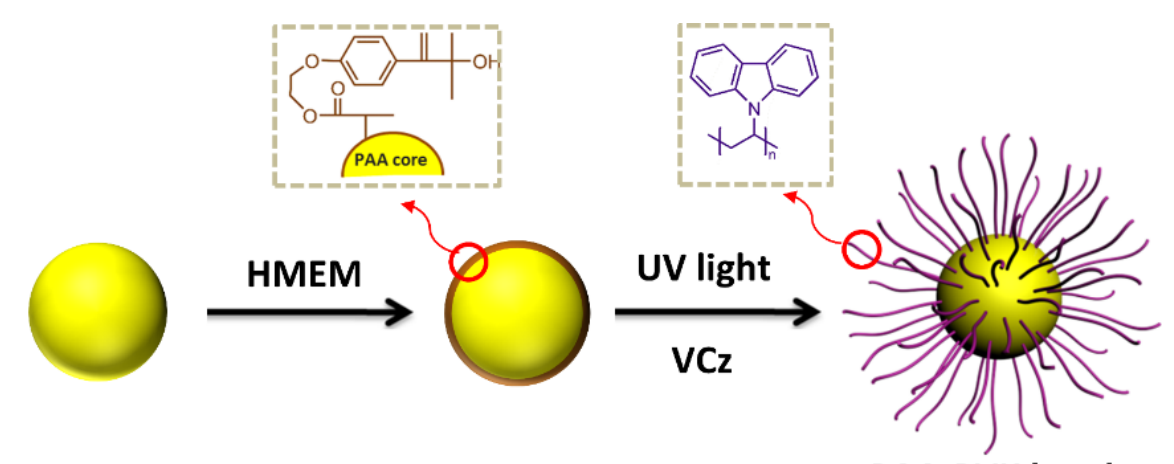

PAA core

PAA@HMEM

PAA-PVK brush

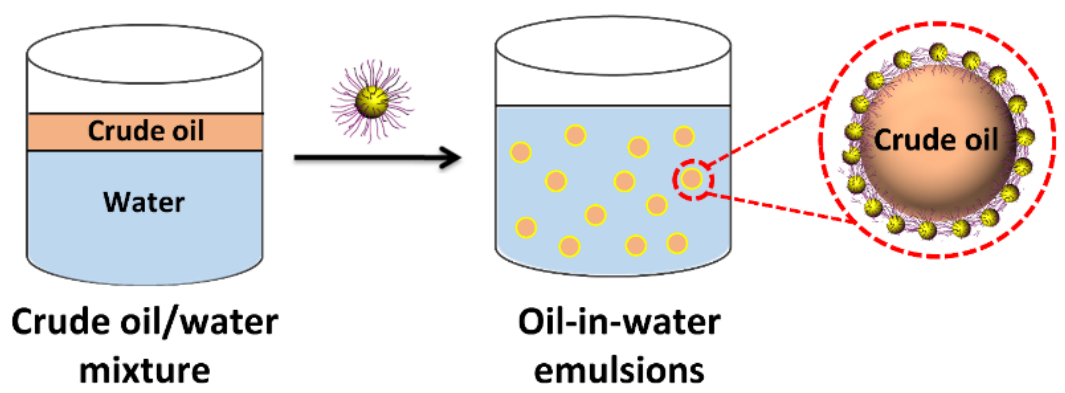

Figure 1. Schematic illustration of PAA-PVK brushes prepared by emulsion polymerization and its applications in crude oil stabilization.
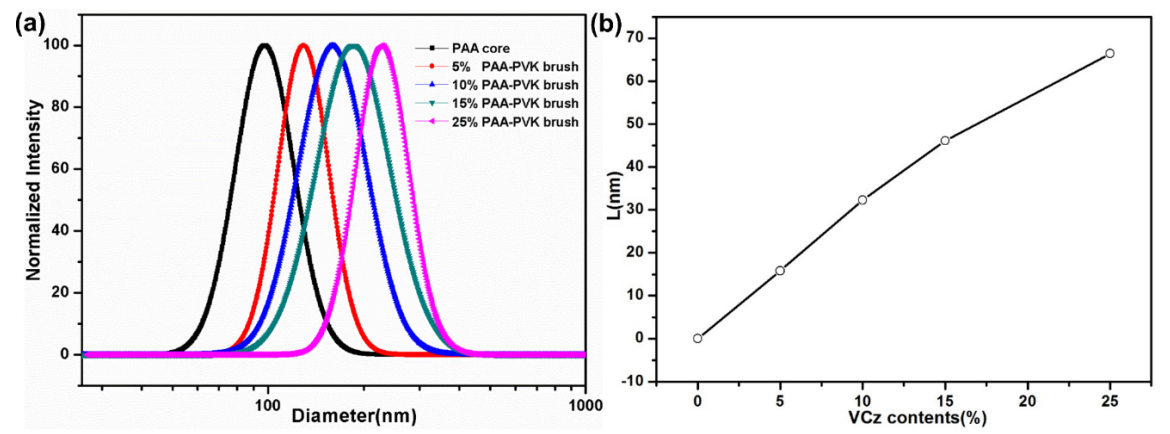

Figure 2. (a) Size and size distribution of PAA-PVK brushes of different VCz content as determined by DLS.(b) effect of $\mathrm{VCz}$ contents on the brush thickness of PAA-PVK brushes. 
(a)

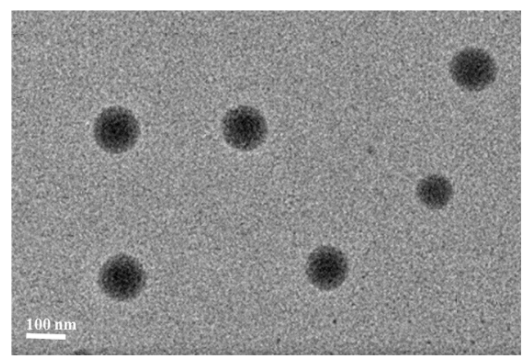

(c)

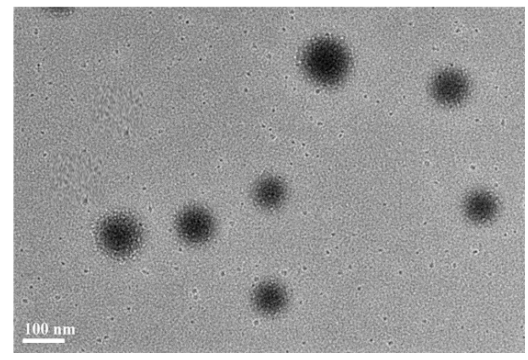

(b)

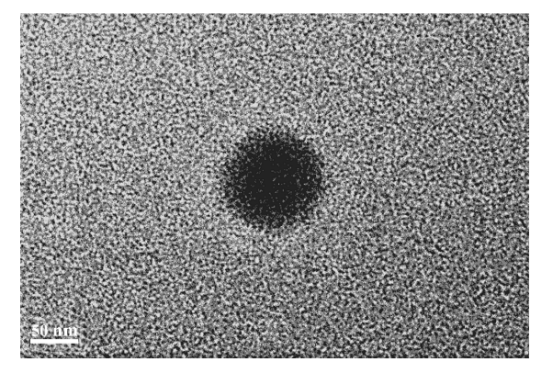

(d)

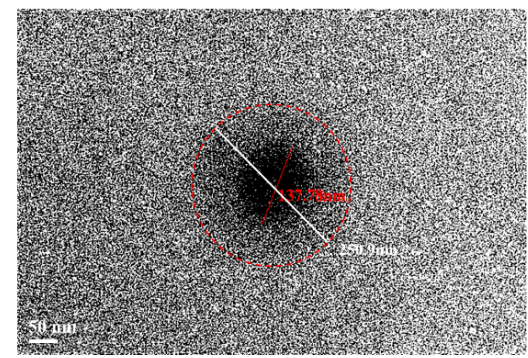

Figure 3. TEM images of PAA core $(\mathrm{a}, \mathrm{b})$ and PAA-PVK brushes (c, d) dispersed in hexane. $\mathrm{VCz}$ content: $25 \%$.
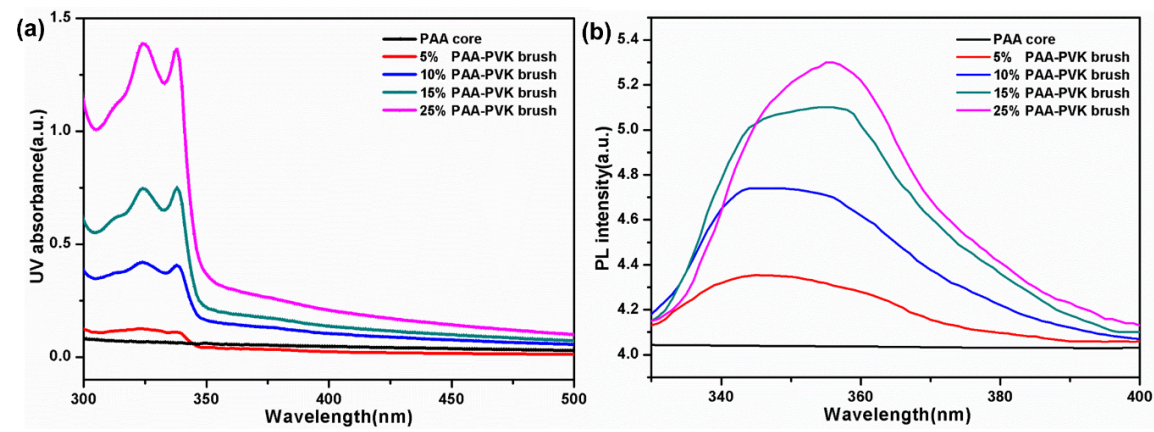

Figure 4. UV-vis (a) and fluorescence (b) spectra of PAA core and PAA-PVK brushes of different VCz content
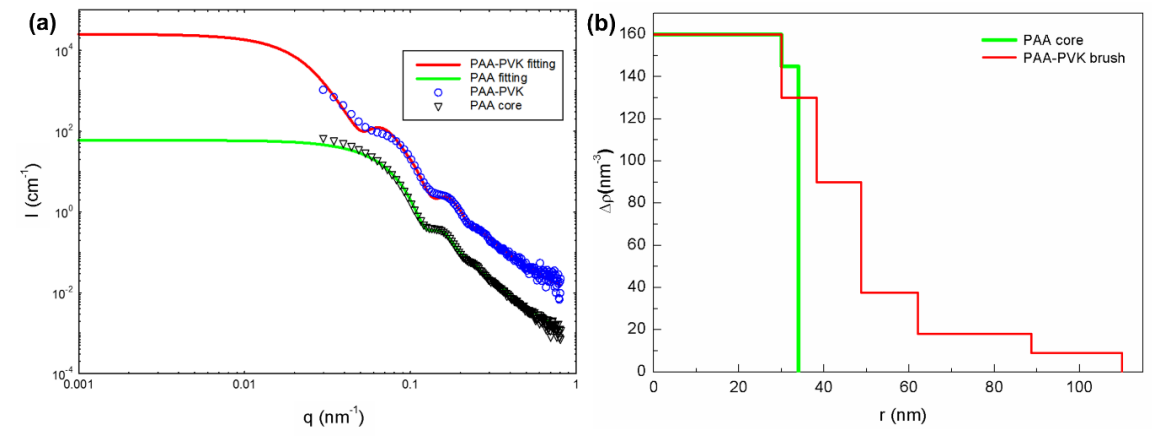

Figure 5. (a) SAXS curves of PAA and PAA-PVK brush. Solid lines represent fitting curves. (b) Excess electron density $\Delta p e(r)$ of core and brush vs. radius. The $\mathrm{VCz}$ content of PAA-PVK brushes:25\% 
(a)

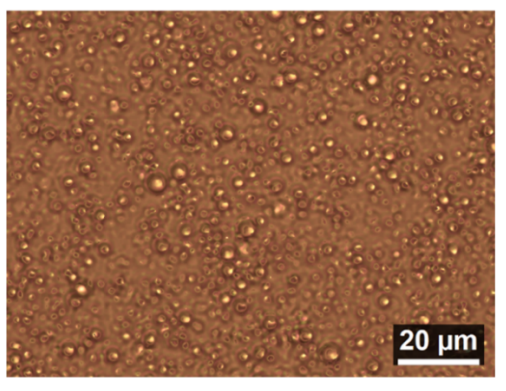

(c)

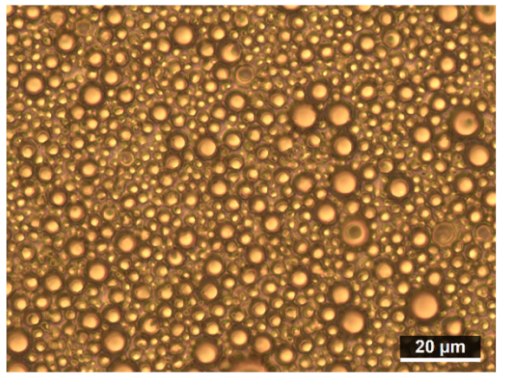

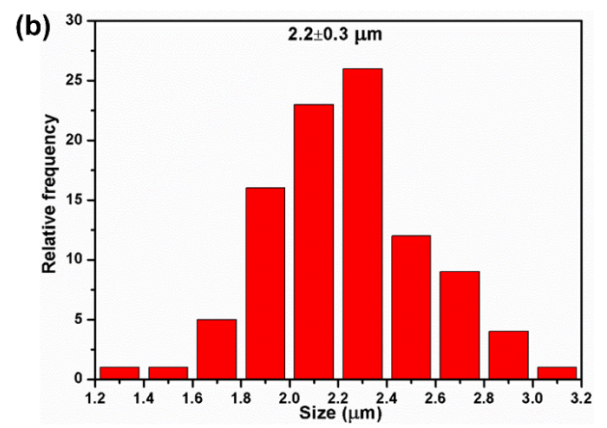

(d)

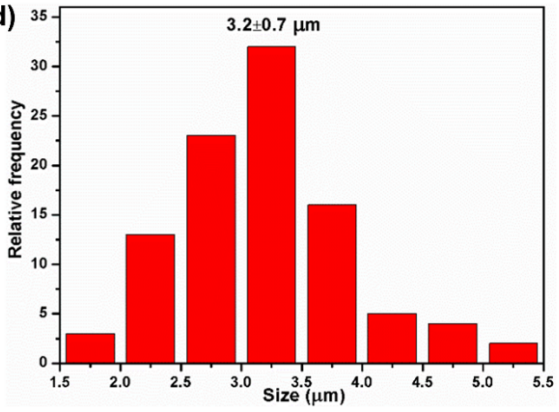

Figure 6. Optical micrographs and particle size analysis of model oil (a, b) and crude oil (c, d) emulsions in water with the presence of PAA-PVK brushes. The solid content for PAA-PVK brushes: $2 \mathrm{mg} / \mathrm{mL}$.
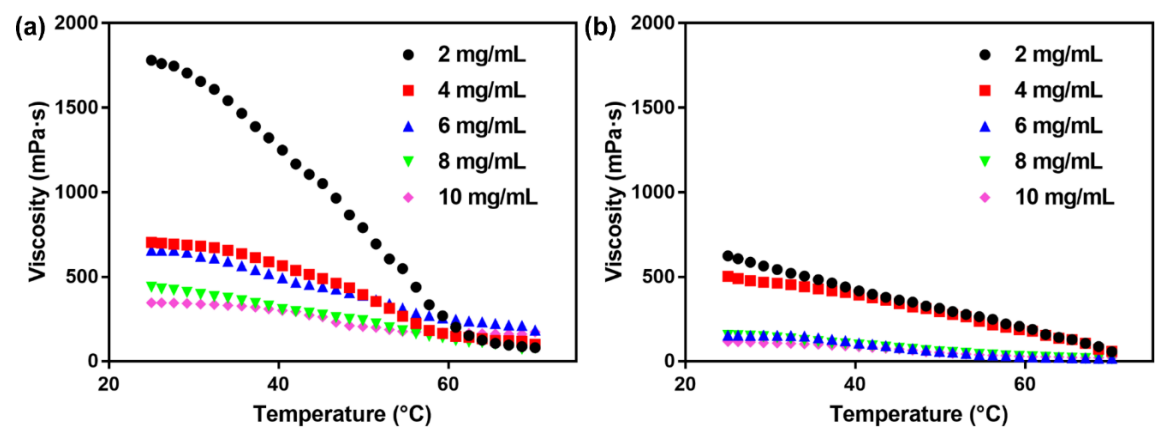

Figure 7. Apparent viscosity of crude oil-in-water emulsions formed by PAA core (a) and PAA-PVK brushes (b) under different concentrations. 


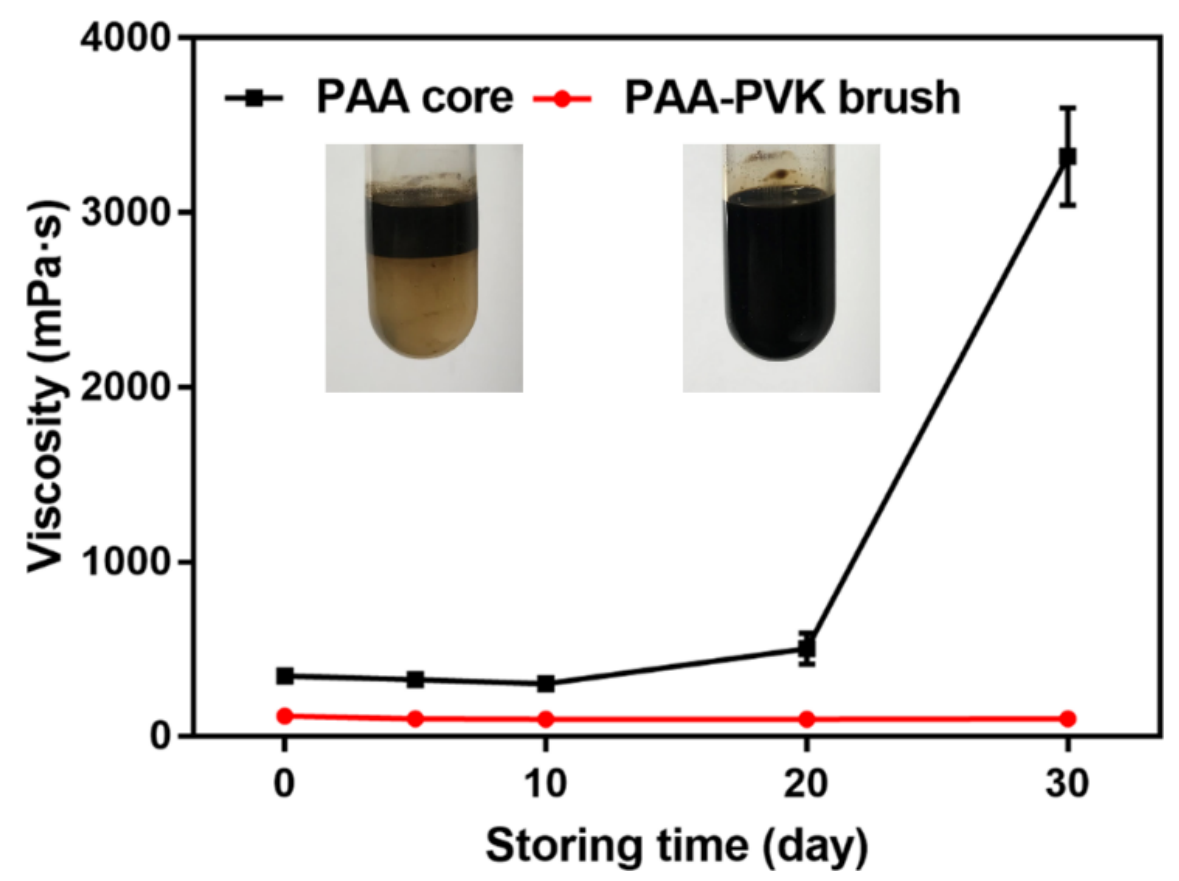

Figure 8. Apparent viscosity of crude oil-in-water emulsions formed by PAA core (black) and PAA-PVK brushes (red) under different storing times. The inset figures are optical images of crude oil-in-water emulsions formed by oil soluble PAA-core (left) and PAA-PVK brushes (right) after storing for 30 days. Solid content for PAA core and PAA-PVK brushes was $2 \mathrm{mg} / \mathrm{mL}$.

\section{Supporting Information}

\section{This material includes}

\section{Experimental}

Properties of Shengli Crude Oil

1. Size and size distribution of PAA core dispersed in hexane.

2. Size variation of PAA core and PAA-PVK brush along with storing time.

3. Size distribution of PAA core and PAA-PVK brush redispersed in different organic media.

4. Optical micrographs and particle size analysis of model oil $(a, b)$ and crude oil $(c, d)$ emulsions in water with the presence of PAA core.

5. Size distribution of water soluble PS-PSS brushes.

6. Optical images of crude oil-in-water emulsions formed by oil soluble PAA-PVK brushes and water soluble PS-PAA brushes.

7. Apparent viscosity of crude oil under different temperatures.

\section{Experimental}

Materials Acrylic acid (AA, Lingfeng Chemical Reagent Co. Ltd.) was purified through reduced pressure distillation to remove polymerization inhibitor and stored in refrigerator in $4^{\circ} \mathrm{C}$ before use. N-Vinylcarbazole ( $\mathrm{VCz}, 98 \%$, Adamas) was purified by recrystallization from methanol. Photoinitiator 2-[p-(2-hydroxy-2methylpropiophenone)]-ethylene glycol-methacrylate (HMEM) and water-soluble PS-PSS spherical polymer brushes were synthesized and used as reported previously ${ }^{[18-20]}$. Span 80 and Tween 80 were purchased from Sinopharm Chemical Regent Co., Ltd. The heavy crude oil sample was kindly donated by Sinopec Shengli Oilfield, and its properties are summarized in Table S1. All other materials of analytical quality were from 
Lingfeng Chemical Reagent Co. Ltd. and used as received. All water used in this work was purified by reverse osmosis and subsequent ion

exchange (Millipore Milli-Q).

Synthesis of PAA latex particles PAA latex particles were prepared through inverse emulsion polymerization based on previous reports [21]. In a typical reaction, $0.75 \mathrm{~g}$ Span 80 and $0.25 \mathrm{~g}$ Tween 80 were mixed in $34 \mathrm{~g}$ hexane as the continuous lipophilic phase and the aqueous phase was prepared by dissolving $5 \mathrm{~g}$ AA in $7.5 \mathrm{~g}$ DI water neutralized by $2.5 \mathrm{~g} \mathrm{NaOH}$. The aqueous phase was added into the oil phase dropwise under mechanical stirring (rate: $300 \mathrm{rpm}$ ) to yield a water-in-oil emulsion. The emulsion was degassed and charged with nitrogen repeatedly to ensure oxygen-free environment. After adjusting the temperature to 50 ${ }^{\circ} \mathrm{C}, 0.0625 \mathrm{~g}$ oil-soluble initiator azodiisobutyronitrile (AIBN) dissolved in $5 \mathrm{~mL}$ toluene was injected into the emulsion. The polymerization reaction lasted for $2.5 \mathrm{~h}$ under nitrogen atmosphere, after which a mixed solution containing $0.2 \mathrm{~g}$ photoinitiator HMEM, $2 \mathrm{~g}$ hexane and $0.5 \mathrm{~g}$ toluene was added at a rate of 6 seconds per drop. An extra $2.5 \mathrm{~h}$ was needed for HMEM to attach to the surface of PAA particles. Finally, the obtained emulsion was filtered to remove possible aggregation and then purified through dialysis against hexane for three days.

Synthesis of polymer brush by photoemulsion polymerization The purified PAA latex particles coated with a thin shell of HMEM were diluted to $1 \mathrm{wt} \%$ with hexane and transferred into a home-made photo reactor. Then grafting monomer $\mathrm{VCz}$, with different amount was added into the reactor and mixed with the latex particles by vigorous magnetic stirring. After repeating evacuation and subsequent charging of nitrogen for three times, the whole reactor was exposed under UV light (wavelength: 200-600 nm, power: $150 \mathrm{~W}$ ) to conduct photo emulsion polymerization for $2.5 \mathrm{~h}$. Finally, the prepared PAA-PVK polymer brush was purified by dialysis against hexane to remove unreacted monomers. The relative monomer content w was used to designate PAA-PVK brushes prepared at different $\mathrm{VCz}$ dose and it could be calculated by the following equation:

$$
w=\frac{m_{\mathrm{VCz}}}{V_{\mathrm{PAA}} x_{\mathrm{PAA}}}
$$

Where $\mathrm{m}_{\mathrm{VCz}}, \mathrm{V}_{\mathrm{PAA}}$ and $\mathrm{x}_{\mathrm{PAA}}$ refers to the mass of $\mathrm{VCz}$ monomer, the volume and solid content of PAA latex particles. In our experiment, PAA-PVK brushes with relative monomer content up to $5 \%, 10 \%, 15$ $\%$ and $25 \%$ was prepared for further application devlopment.

Preparation of Oil-in-Water $(\mathrm{O} / \mathrm{W})$ emulsions The PAA core and PAA-PVK brushes of $25 \%$ monomer content (solid content: $250 \mathrm{mg} / \mathrm{mL}$ ) was chosen as the oil-in-water emulsifier. The oil-in-water emulsions were generated by adding $1 \mathrm{~mL}$ obtained latex particles into $25 \mathrm{~mL}$ of the mixture of oil and water and then homogenizing for certain times. For model oil, the emulsion was prepared by sonication for 5 minutes with the volume ratio of water/decane set as $3: 7.53 \mathrm{KHz}$ and $350 \mathrm{~W}$ were chosen as the sonication parameters by employing a sonicator (SK7210LHC from KUDOS Co.) in the entire experiments. For crude oil, the emulsion was prepared by homogenizing with a mechanical stirrer Ultra-Turrax T25 for 5 minutes (15000 $\mathrm{rpm}$ ) with the mass ratio of water/crude oil set as 5:1. The dispersion state and morphology of oil-in-water emulsions was observed by polarizing optical microscopy (Leica DM2500 P microscope) and the apparent viscosity of the crude oil emulsions was recorded by rheometer (Anton Paar, MCR 501).

Characterizations Dynamic light scattering (DLS) was carried out using a particle sizing system, NICOMP 380 ZLS equipped with a scattering angle of $90^{\circ}$. Transmission electron microscopy (TEM) was performed by a JEOL JME-1400 transmission electron microscope at acceleration voltage of $100 \mathrm{kV}$. TEM samples were prepared by adding one drop of particle solution on a copper grid (300 mesh) and then dried at ambient environment overnight. The optical properties of PAA-PVK brushes were characterized by UV-3250 UV-Vis spectrophotometer, (SHIMADZU) and RF-5301PC fluorometer (SHIMADZU). SAXS measurements were performed at BL16B1 beamline in Shanghai Synchrotron Radiation Facility (SSRF, China). The detector used was MAR165 CCD detector and the sample-to-detector distances were set as $5 \mathrm{~m}$. During each SAXS 
measurement, 0.1-0.2 mL solution was added into a sample cell by syringe and the sample cell was a 1-mmthick polystyrene plastic template wrapped by polyimide film on both sides. The morphology of emulsion droplets was observed with a Leica DM2500 P microscope. A small drop of emulsion was placed on a microscope slide for observation and images were captured with a charge-coupled device camera connected to a computer via WT-1000GM imaging board. Software ImageJ was then utilized to analyze the average size and size distribution of the formed emulsions. The apparent viscosity of crude oil emulsions in different temperatures was tested by Anton Paar MCR 501 rheometer with 30 points recorded at a interval of 10 s. The shear rate was set as $100 \mathrm{~s}^{-1}$ and apparent viscosity is recorded at temperature range of $25-70{ }^{\circ} \mathrm{C}$.

Table S1. Properties of Shengli Crude Oil.

\begin{tabular}{lllllll}
\hline Property & $\begin{array}{l}\text { Viscosity } \\
(\mathrm{mPa} \cdot \mathrm{s})\end{array}$ & $\begin{array}{l}\text { Density } \\
\left(\mathrm{g} \cdot \mathrm{cm}^{-3}\right)\end{array}$ & $\begin{array}{l}\text { Saturates } \\
(\mathrm{wt} \%)\end{array}$ & $\begin{array}{l}\text { Aromatic } \\
(\mathrm{wt} \%)\end{array}$ & $\begin{array}{l}\text { Asphaltenes } \\
(\mathrm{wt} \%)\end{array}$ & $\begin{array}{l}\text { Resins }(\mathrm{wt} \%) \\
\text { Value }\end{array}$ \\
\hline
\end{tabular}

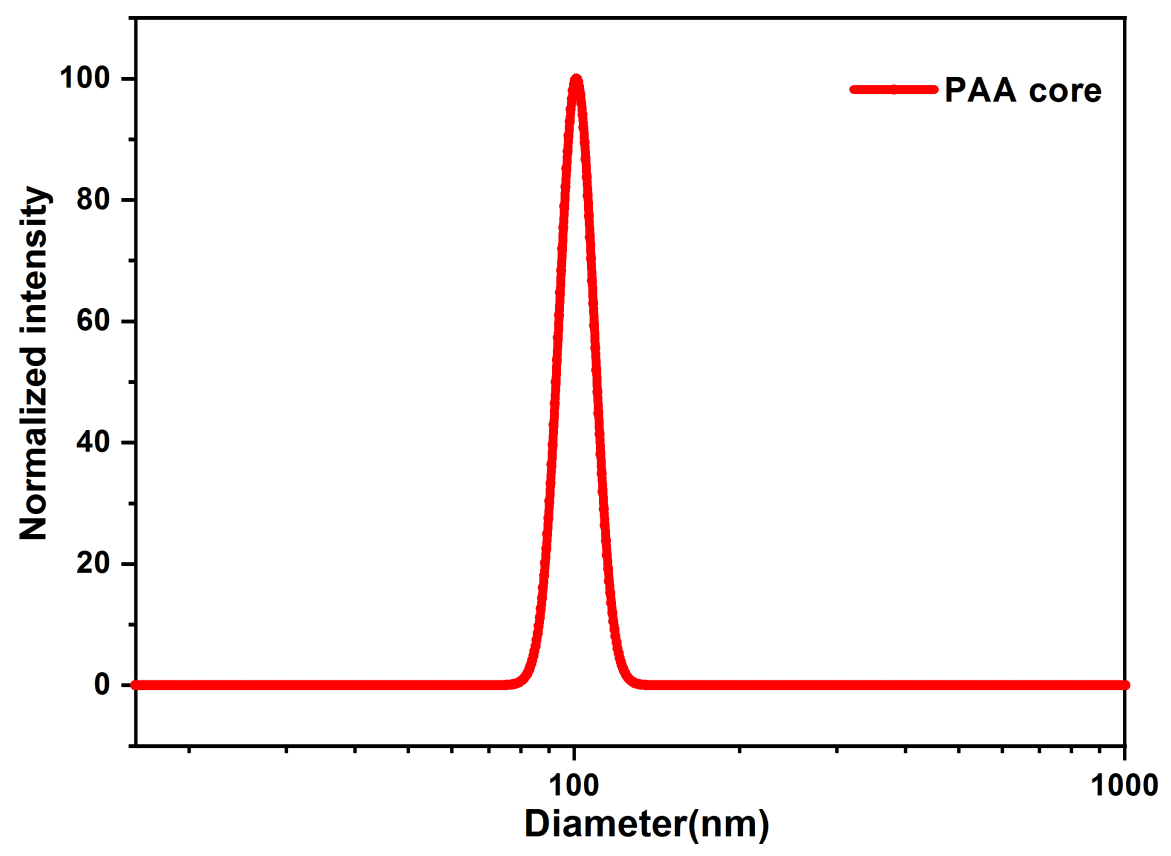

Figure S1. Size and size distribution of PAA core dispersed in hexane. 


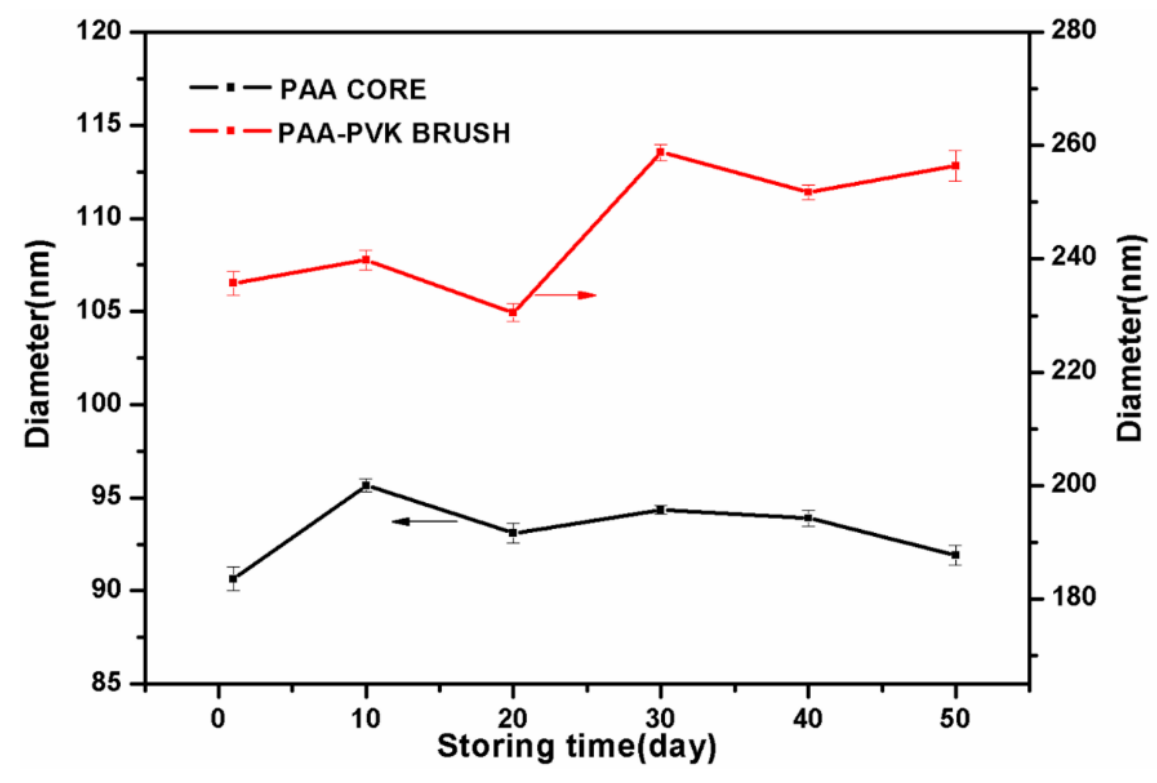

Figure S2. Size variation of PAA core and PAA-PVK brush along with storing time. VCz content: $25 \%$.

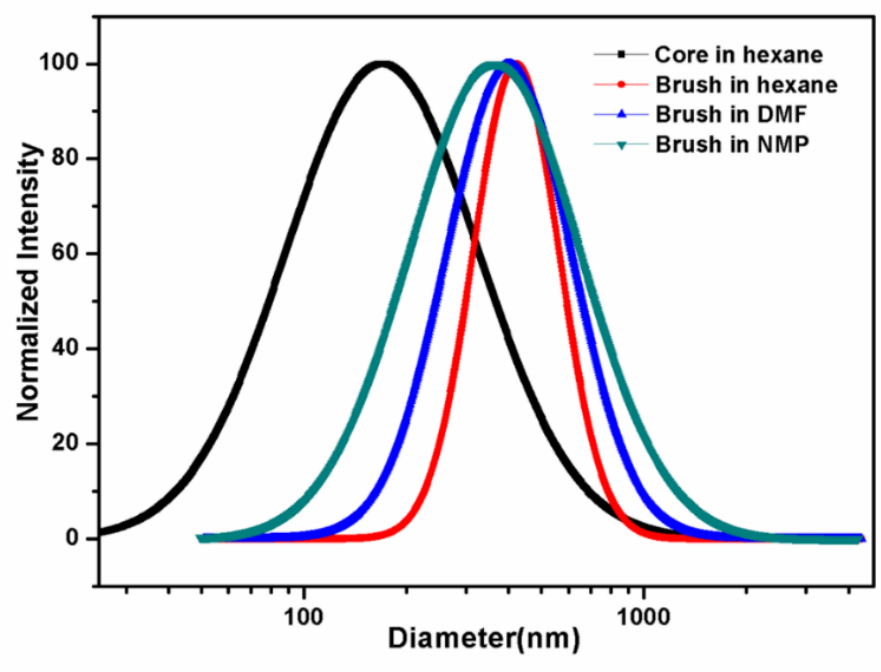

Figure S3. Size distribution of PAA core and PAA-PVK brush redispersed in different organic media. VCz content:25\%. 
(a)

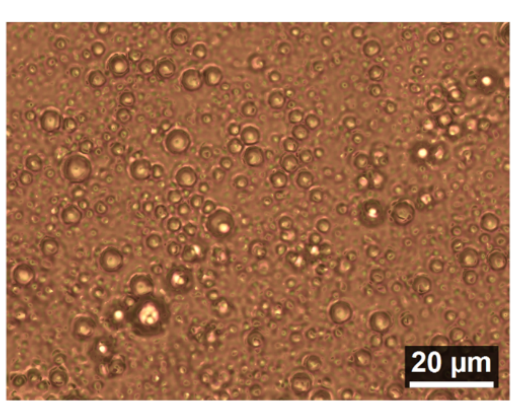

(c)

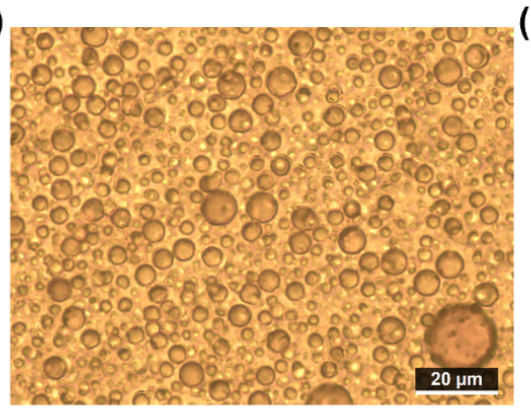

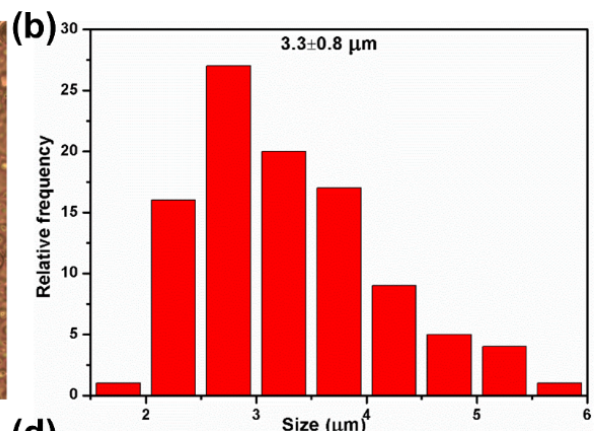

(d)

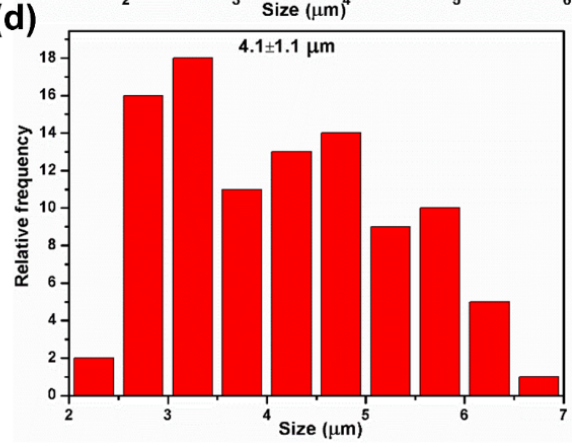

Figure S4. Optical micrographs and particle size analysis of model oil $(a, b)$ and crude oil (c, d) emulsions in water with the presence of PAA core. The solid content for PAA cores: $2 \mathrm{mg} / \mathrm{mL}$.

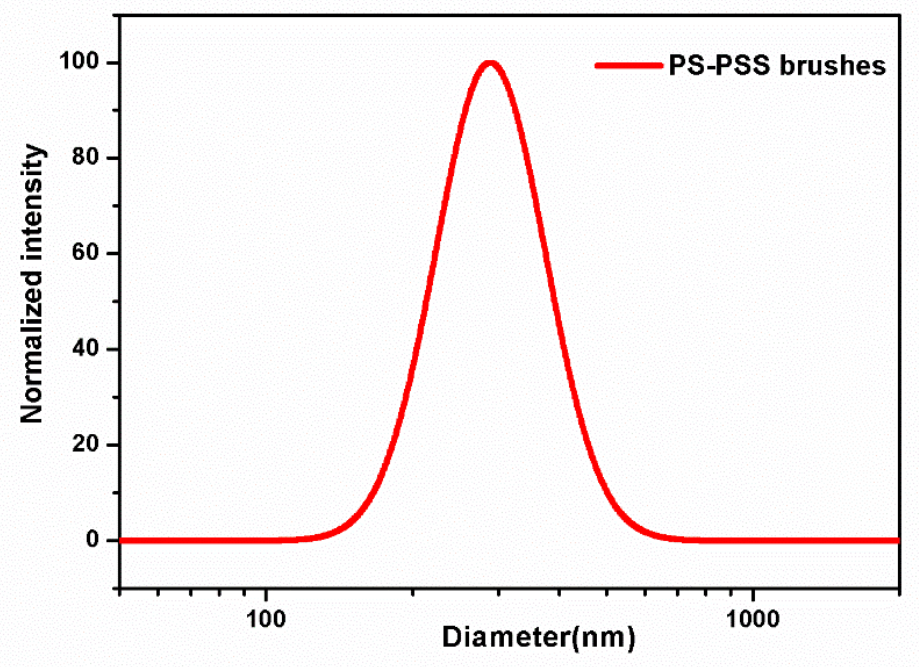

Figure S5. Size distribution of water soluble PS-PSS brushes.SS content: $25 \%$. 


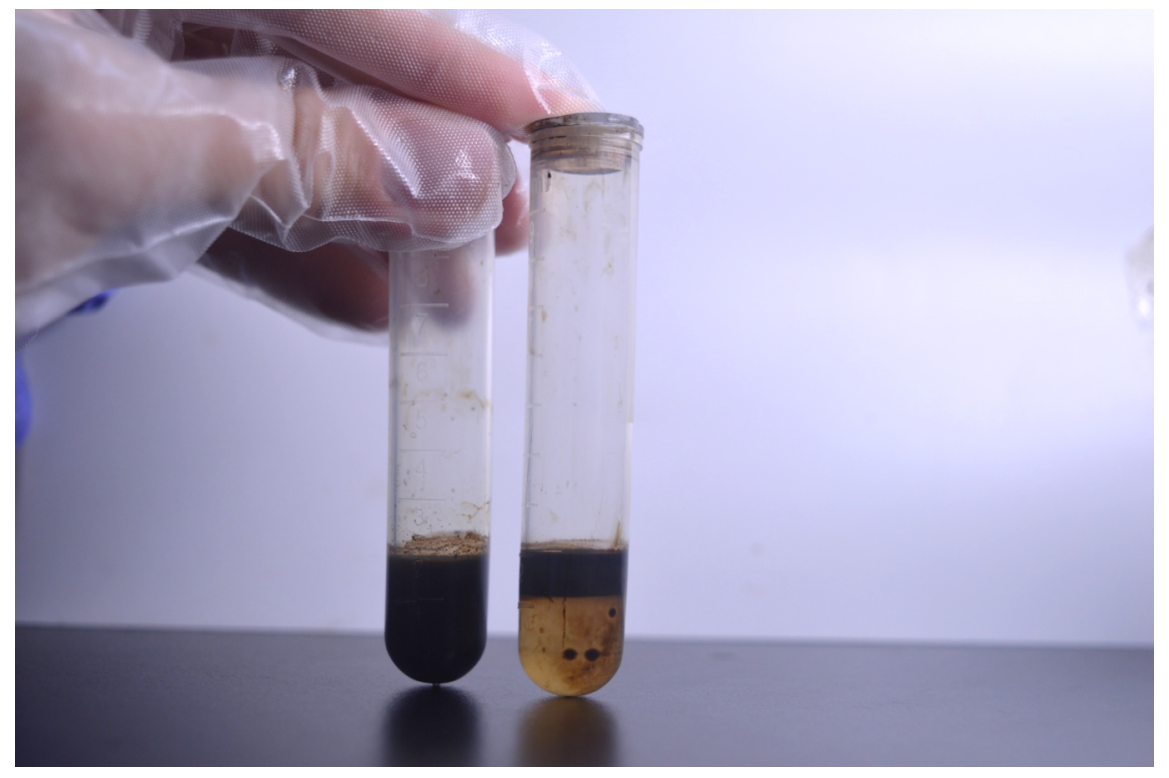

Figure S6. Optical images of crude oil-in-water emulsions formed by oil soluble PAA-PVK brushes (left) and water soluble PS-PAA brushes (right). Solid content for brushes content: 2 $\mathrm{mg} / \mathrm{mL}$.

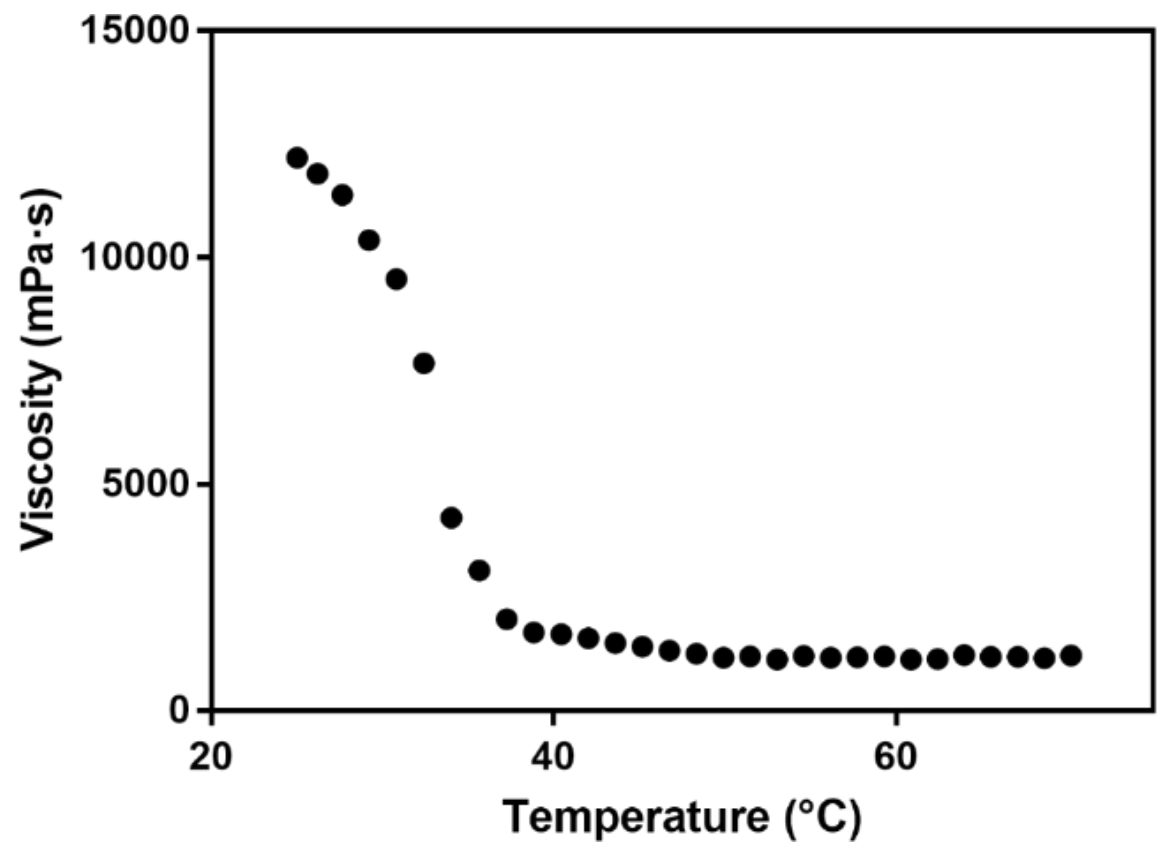

Figure S7. Apparent viscosity of crude oil under different temperatures. 